\title{
Formation of Two-Neutron Halo in Light Drip-Line Nuclei from the Low-Energy Neutron-Neutron Interaction
}

\author{
Toshio Suzuki ${ }^{1}$ \\ Department of Physics, College of Humanities and Sciences, Nihon University \\ Sakurajosui 3-25-40, Setagaya-ku, Tokyo 156-8550, Japan \\ E-mail: suzuki@phys.chs.nihon-u.ac.jp \\ Takaharu Otuska \\ Department of Physics, the University of Tokyo \\ Hongo 7-3-1, Bunkyo-ku, Tokyo 113-0033, Japan \\ E-mail: otsuka@phys.s.u-tokyo.ac.jp
}

\section{Cenxi Yuan}

Sino-French Institute of Nuclear Engineering and Technology, Sun Yat-Sen University

Zhuhai, 519082, Guangdong, China

E-mail: yuancx@mail.sysu.edu.cn

\section{Navin Alahari}

GANIL, CEA/DSM-CNRS/IN2P3

Bd Henri Becquerel, BP 55027, F-14076, Caen Cedex 5, France

E-mail: navin@ganil.fr

The formation of two-neutron halo is described using the neutron-neutron (n-n) interaction fixed at the low-energy $n-n$ scattering limit. This method is tested for loosely-bound two neutrons in ${ }^{24} \mathrm{O}$, where a good agreement with experimental data is found. It is applied to halo neutrons in ${ }^{22} \mathrm{C}$ in two ways: with the ${ }^{20} \mathrm{C}$ core being closed or correlated due to excitations from the closed core. This nn interaction is shown to be strong enough to produce a two-neutron halo in both cases, locating ${ }^{22} \mathrm{C}$ on the drip line, while ${ }^{21} \mathrm{C}$ remains unbound. A unique relation between the two neutron separation energy, $\mathrm{S}_{2 \mathrm{n}}$, and the radius of neutron halo is presented. New predictions for $S_{2 n}$ and the radius of neutron halo are given for ${ }^{22} \mathrm{C}$. The estimated halo radius is found to be consistent with a recent experimental data. The appearance of Efimov states is also discussed. Spectra of excited states in ${ }^{22} \mathrm{C}$ are predicted. The $\mathrm{n}-\mathrm{n}$ interaction used here is large compared to conventional shell-model interactions. Roles of three-body forces are discussed.

The 26th International Nuclear Physics Conference

11-16 September, 2016

Adelaide, Australia

\footnotetext{
${ }^{1}$ Speaker

(C) Copyright owned by the author(s) under the terms of the Creative Commons 


\section{Introduction}

Two-neutron halos are found in several light drip-line nuclei such as ${ }^{10} \mathrm{He},{ }^{14} \mathrm{Be},{ }^{19} \mathrm{~B}$ and ${ }^{22} \mathrm{C}$ since it was found in ${ }^{11} \mathrm{Li}[1]$. When one neutron is removed from these nuclei, they become unbound. The two-neutron halos in ${ }^{11} \mathrm{Li},{ }^{14} \mathrm{Be}$ and ${ }^{19} \mathrm{~B}$ have rather complex structure, that is, with mixtures of two or more orbits, but those in ${ }^{10} \mathrm{He}$ and ${ }^{22} \mathrm{C}$ have simple structure with almost pure $\mathrm{s}_{1 / 2}^{2}$ components. Here, we are mainly concerned with the halo structure of ${ }^{22} \mathrm{C}$. We study how the two-neutron halo in ${ }^{22} \mathrm{C}$ is formed from the low energy limit of neutron-neutron (n-n) interaction. We use a three-body model which assumes a ${ }^{20} \mathrm{C}$-core and two valence neutrons interacting each other. This is a Borromean situation [2,3], and similar configurations have been considered in previous theoretical works [4-7] but with different methods and interactions. The ${ }^{20} \mathrm{C}$-core is treated, on one hand, as a closed-core with $1 \mathrm{p}^{10} v 1 \mathrm{~d}_{5 / 2}{ }^{6}$ configurations, and on the other hand as a correlated-core with mixing of $2 \mathrm{~s}_{1 / 2}$ orbit. ${ }^{20} \mathrm{C}$ is known to have $2 \mathrm{~s}_{1 / 2}{ }^{2}$ components whose spectroscopic factor is about 1 [8]. Relations between the two-neutron separation energy, $\mathrm{S}_{2 \mathrm{n}}$, and the halo radius are derived for both cases. The halo radius is estimated by taking into account a condition for ${ }^{21} \mathrm{C}$ to be unbound, and compared with experimental data. A comment on Efimov states is made. In sect. 2, a three-body model is explained and applied to ${ }^{24} \mathrm{O} . \mathrm{S}_{2 \mathrm{n}}$ and halo radius in ${ }^{22} \mathrm{C}$ are studied using both closed-shell and correlated ${ }^{20} \mathrm{C}$-core. Spectra of ${ }^{22} \mathrm{C}$ are also predicted. In sect. 3, the low energy bare $\mathrm{n}-\mathrm{n}$ interaction is compared with $\mathrm{n}-\mathrm{n}$ interaction in the medium, and roles of there-body forces are investigated. A summary is given in sect. 4 .

\section{Three-body model with low energy n-n intearction}

We use a three-body model consisiting a core and two neutrons. Neutron-core interaction is taken to be a one-body Woods-Saxon (WS) potential. The energy of a neutron in the WS potential,$\varepsilon$, is positive (negative) if it is unbound (bound). For the $n-n$ interaction, $\mathrm{v}_{\mathrm{n}}$, one in the low energy limit which reproduces both the scattering length, $a_{n n}$, and the effective range, $r_{n n}$, is used [9]. A gaussian radial form with the range of $1.795 \mathrm{fm}$ is adopted, whose strength is adjusted to give $a_{n n}=-18.9 \mathrm{fm}$ and $r_{n n}=2.75 \mathrm{fm}$. The two-neutron energy, $E_{n n}$, is given by

$$
\mathrm{E}_{\mathrm{nn}}=2 \varepsilon+<\mathrm{v}_{\mathrm{nn}}>
$$

where $\left\langle\mathrm{v}_{\mathrm{nn}}\right\rangle$ is the matrix element of $\mathrm{v}_{\mathrm{nn}}$ for the two-neutron wave function and is negative. The three-body system is bound if $E_{n n}$ is negative. Two-neutron separation energy is defined by $S_{2 n}=$ $-E_{n n}$, and as one-neutron separation energy is given by $\left.S_{1 n}=-\left(\varepsilon+<v_{n n}\right\rangle\right), S_{2 n}=2 S_{1 n}+\left\langle v_{n n}\right\rangle$. Note that $S_{1 n}=\left(S_{2 n}-\left\langle v_{n n}\right\rangle\right) / 2$ is larger than $S_{2 n} / 2$. The halo wave function damps more rapidly than that obtained with $S_{1 \mathrm{n}}=\mathrm{S}_{2 \mathrm{n}} / 2$.

Here, for a neutron the attraction from the other neutron is treated as an aditional onebody potential obtained by convolution of $\mathrm{v}_{\mathrm{nn}}$ by the neutron wave functions. $\mathrm{S}_{1 \mathrm{n}}$ and bound neutron wave function are obtained by solving a Schrodinger equation in the WS+ the additional potential. The neutron wave function is used for the convolution of $\mathrm{v}_{\mathrm{nn}}$ again. Thus, after this iterative procedure, $S_{1 n},\left\langle v_{n n}\right\rangle$ and $S_{2 n}$ are obtained self-consistently when they are converged.

\subsection{Application to ${ }^{24} \mathrm{O}$}

In the three-body model, ${ }^{24} \mathrm{O}$ is treated as ${ }^{22} \mathrm{O}$-core and two neutrons. ${ }^{23} \mathrm{O}$ is bound with $\varepsilon=$ $-2.73 \mathrm{MeV}$. The WS potential is fixed to reproduce this value for $\varepsilon$. Then, $S_{2 n}$ and $S_{1 n}$ are 
obtained by the above procedure; $S_{2 n}=6.94 \mathrm{MeV}$ and $S_{1 n}=4.21 \mathrm{MeV}$ which are very close to the experimental values of $S_{2 n}=6.92 \mathrm{MeV}$ and $S_{1 n}=4.19 \mathrm{MeV}$, respectively [10].

Next, we discuss matter radii of the oxygen isotopes. Calculated root-mean-square (r.m.s.) matter radii of ${ }^{22} \mathrm{O},{ }^{23} \mathrm{O}$ and ${ }^{24} \mathrm{O}$ in the present three-body model are shown in Table I as well as the experimental values $[11,12]$. Here, except for the neutron $2 s_{1 / 2}$ orbit harmonic oscillator wave functions are used for the evaluation of the matter radii with the center-of-mass corrections. The calculated matter radii are found to be close to the experimental values. We thus find that the present three-body model is quite successfull fo rthe description of the dripline nucleus ${ }^{24} \mathrm{O}$.

\begin{tabular}{|l|l|l|l|}
\hline Isotopes & $\begin{array}{l}\text { Present three- } \\
\text { body model }\end{array}$ & Ozawa [11] & Kanungo [12] \\
\hline${ }^{22} \mathrm{O}$ & 2.85 & $2.88 \pm 0.06$ & $2.75 \pm 0.15$ \\
\hline${ }^{23} \mathrm{O}$ & 2.97 & $3.20 \pm 0.04$ & $2.95 \pm 0.23$ \\
\hline${ }^{24} \mathrm{O}$ & 3.03 & $3.19 \pm 0.13$ & \\
\hline
\end{tabular}

Table I Root-mean-square matter radii of oxygen isotopes in units of fm

\section{$2.2{ }^{22} \mathrm{C}$ with closed-shell ${ }^{20} \mathrm{C}$-core}

We now discuss ${ }^{22} \mathrm{C}$ which consisits of ${ }^{20} \mathrm{C}$-core and two neutrons. We first assume that ${ }^{20} \mathrm{C}$ has a simple closed-core configuration with $1 \mathrm{p}^{10} v 1 \mathrm{~d}_{5 / 2}{ }^{6}\left(0^{+}\right)$. As ${ }^{21} \mathrm{C}$ is unbound $\varepsilon$ is positive. The attractive $n-n$ interaction makes ${ }^{22} \mathrm{C}$ bound. For each value of the strength of the WS potential, $\mathrm{S}_{2 \mathrm{n}}, \mathrm{V}_{\mathrm{nn}}>, \mathrm{S}_{1 \mathrm{n}}$ and neutron halo wave function are obtained self-consisitently. The relation between $S_{2 n}$ and the r.m.s. radius of the halo, $\left\langle r^{2}\right\rangle^{1 / 2}$, is shown in Fig. 1. Here, the case for $S_{1 n}=S_{2 n} / 2$ is also shown. Experimetal range for $S_{2 n}$ are denoted by vertical lines [10] and possible values by arrows [8]. Another experiment suggests a value of $S_{2 n}=-0.140 \pm 0.460$ $\mathrm{MeV}$ [13]. Experimental value for $\mathrm{S}_{2 \mathrm{n}}$ is rather scatterd and it is not well determined. As $\varepsilon=-$ $\left(\mathrm{S}_{2 \mathrm{n}}+\left\langle\mathrm{v}_{\mathrm{nn}}\right\rangle\right) / 2$, the condition for ${ }^{21} \mathrm{C}$ to be unbound is $\left.\varepsilon\right\rangle 0$, that is, $\mathrm{S}_{2 \mathrm{n}}\left\langle-\left\langle\mathrm{v}_{\mathrm{nn}}\right\rangle=\left|\left\langle\mathrm{v}_{\mathrm{nn}}\right\rangle\right|\right.$. This condition is satisfied for $\mathrm{S}_{2 \mathrm{n}}<0.3 \mathrm{MeV}$. As for r.m.s. radius of the neutron halo, a value of $15.97+3.67 /-3.97 \mathrm{fm}$ was obtained from proton-carbon scattering experiment [14]. This value looks to be consistent with the present model with the closed-shell ${ }^{20} \mathrm{C}$-core for $\mathrm{S}_{2 \mathrm{n}} \leqq 110 \mathrm{keV}$. But, this is not the end of the story.

\section{3 $\quad{ }^{22} \mathrm{C}$ with correlaed ${ }^{20} \mathrm{C}$-core}

We next study the case for correlated ${ }^{20} \mathrm{C}$. Occupation number of neutron $2 \mathrm{~s}_{1 / 2}$ orbit is about 1[8]. It is also true for a shell-model calculation with the YSOX interaction [15]. There is a large mixing of components of $v 1 \mathrm{~d}_{5 / 2}{ }^{4} 2 \mathrm{~s}_{1 / 2}{ }^{2}$ configurations, which lowers the ground state energy of ${ }^{20} \mathrm{C}$. On the other hand, the halo state in ${ }^{22} \mathrm{C}$ is dominantly $2 \mathrm{~s}_{1 / 2}{ }^{2}$ configuration [8]. How to reconcile this situation?

We here adopt the following model. Halo s-orbit is occupied by two neutrons as predicted by the experiments [8]. Orthogonality condition between this halo s-orbit and the s-orbit of the ${ }^{20} \mathrm{C}$-core state should be satisfied, that is, the core s-orbit is made orthogonal to this halo s-orbit by Gram-Schmidt method. This gives rise to blocking effects on the core states. Energy of the 
${ }^{20} \mathrm{C}$-core of the ground state of ${ }^{22} \mathrm{C}$ is shifted with respect to the energy of the ${ }^{20} \mathrm{C}$ ground state. This energy shift will be denoted as $\Delta$, which should be positive due to the blocking of the $1 \mathrm{~d}_{5 / 2}^{4} 2 \mathrm{~s}_{1 / 2}{ }^{2}$ configurations. The two-neutron separation energy is thus reduced; $\mathrm{S}_{2 \mathrm{n}}=-\mathrm{E}_{\mathrm{nn}}-\Delta$.

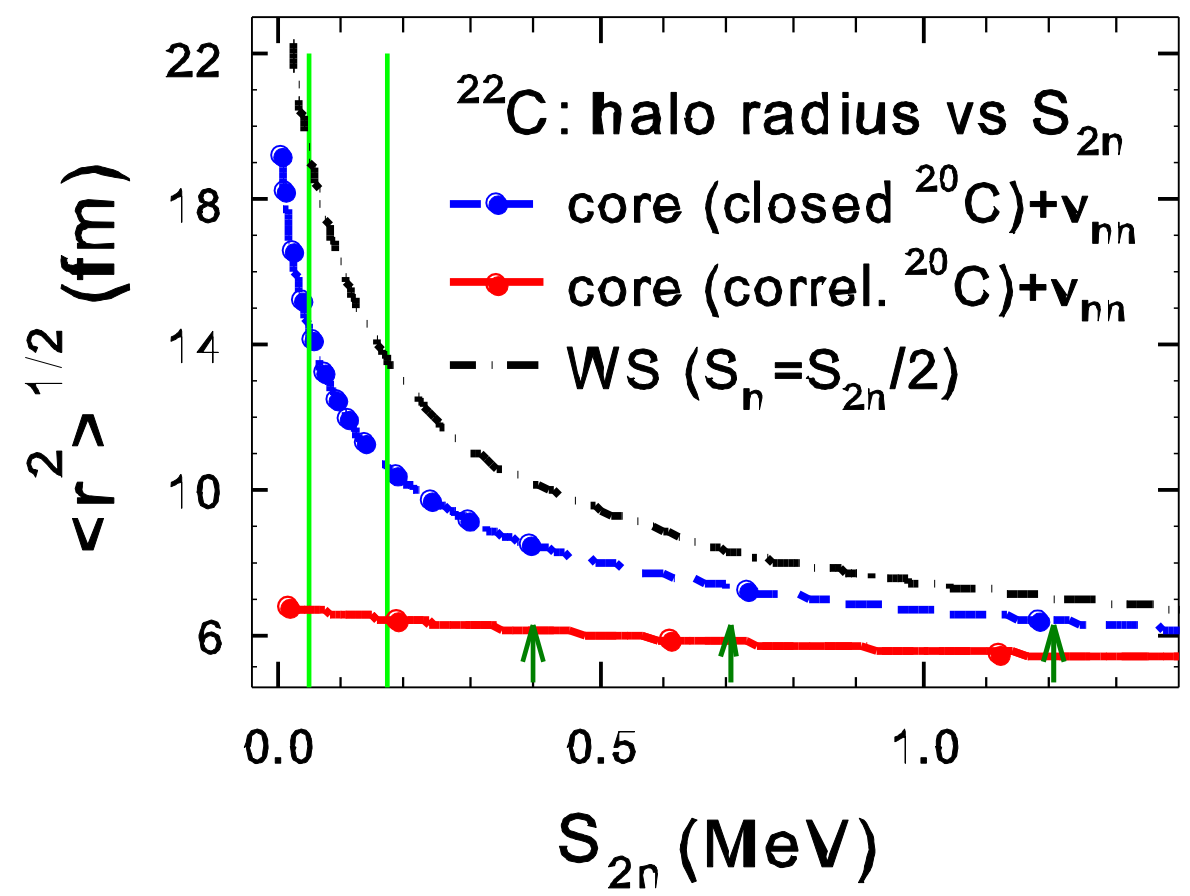

Fig. 1 R.m.s. radius of the halo neutron as a function of two-neutron separation energy $S_{2 n}$. Blue dashed line and filled circles indicate the result obtained with the core of the closed-shell ${ }^{20} \mathrm{C}$, while red solid line and filled circles the result of the core of correlated ${ }^{20} \mathrm{C}$. The result obtained from WS potential $\left(S_{1 n}=S_{2 n} / 2\right)$ without $v_{n n}$ is shown by the black dash-dotted line. The range of $S_{2 n}$ obtained from Ref. [10] is shown by green vertical lines. Green arrows denote values discussed in [8] . (Taken from Fig. 5 of Suzuki et al., Phys. Lett. B753 (2016) 199 [16].)

Halo and core $s$ states are defined as

$$
\begin{aligned}
& \left.|\tilde{\mathrm{s}}\rangle=\mid \mathrm{s}_{1 / 2}(\text { halo })>=\alpha \mid 2 \mathrm{~s}_{1 / 2}(\text { H.O. })>+\beta \mid \text { far-s }\right\rangle \\
& |\overline{\mathrm{s}}\rangle=\mid \mathrm{s}_{1 / 2}(\text { core })>=\beta \mid 2 \mathrm{~s}_{1 / 2}(\text { H.O. })>-\alpha \mid \text { far-s }>
\end{aligned}
$$

with $\langle\overline{\mathrm{s}} \mid \tilde{\mathrm{s}}\rangle=0$. In Eq. (2) $\mid$ far-s $\rangle$ denotes the halo part of the neutron wave function whose tail extends far away from the center. For deeply bound states, $\alpha \rightarrow 1$ and $\beta \rightarrow 0$ with less $2 \mathrm{~s}_{1 / 2}$ component in $\left|\mathrm{s}^{-}\right\rangle$. The ground state energy of ${ }^{20} \mathrm{C}$ is pushed up with $\Delta>0$. As $\mid \mathrm{s}_{1 / 2}$ (core) $>$ gets halo components, two-body matrix elements of the YSOX interaction with s-components are modified. The single-particle energy of $2 \mathrm{~s}_{1 / 2}$ orbit outside the ${ }^{4} \mathrm{He}$-core is also modified. Shellmodel calculations are performed by taking into account these modifications in configurations with protons in the p-shell and neutrons in the sd-shell. The energy shift of the ground state of ${ }^{20} \mathrm{C}$ is evaluated to be $\Delta \sim 1 \mathrm{MeV}$.

The relation between $\mathrm{S}_{2 \mathrm{n}}$ and the halo radius for the correlated ${ }^{20} \mathrm{C}$-core case is shown in Fig. 1 (red solid line). The condition for ${ }^{21} \mathrm{C}$ to be unbound gives $S_{2 n}<0.8 \mathrm{MeV}$. Fig. 1 suggests 
that the r.m.s. radius of the neutron halo is 6-7 fm, which is quite small compared with the value obtained in Ref. [14]. The amount of the $2 \mathrm{~s}_{1 / 2}$ component in $\mid \mathrm{s}_{1 / 2}$ (core) $\rangle$ state is $\beta^{2} \sim 40-50 \%$. Recently, the matter radius of ${ }^{22} \mathrm{C}$ was re-measured, which yields $3.44 \pm 0.08 \mathrm{fm}$ [17] much smaller compared with the previous one, $5.4 \pm 0.9 \mathrm{fm}[14]$. A smaller halo radius of $6.74+0.71 /-$ 0.48 , which is consistent with the present three-body model with the correlated ${ }^{20} \mathrm{C}$-core, is estimated from the recent measurement [17] and the radius of ${ }^{20} \mathrm{C}$ [11]. As dependence of the halo radius on $S_{2 n}$ is small, it is difficult to determine the value of $S_{2 n}$ from the radius.

The hypothesis of Efimov [18,19] states implies the appearance of similar states at different scales near threshold. The upper bound on the radius of the halo in the correlated-core model contradicts this hypothesis. The ground state of ${ }^{22} \mathrm{C}$ is already close to this upper limit, and there are no excited bound states. The state of the two-neutron halo in ${ }^{22} \mathrm{C}$ can be called a single Efimov state for the correlated-core case.

\subsection{Spectra of ${ }^{22} \mathrm{C}$}

We discuss energy spectra of ${ }^{22} \mathrm{C}$. Shell-model calculations with the modified two-body YSOX interaction and the modified single-particle energy of the $2 \mathrm{~s}_{1 / 2}$ orbit are carried out for the correlated-core cases. Calculated spectra are shown in Fig. 2 as well as for the original YSOX Hamiltonian without the halo effects.

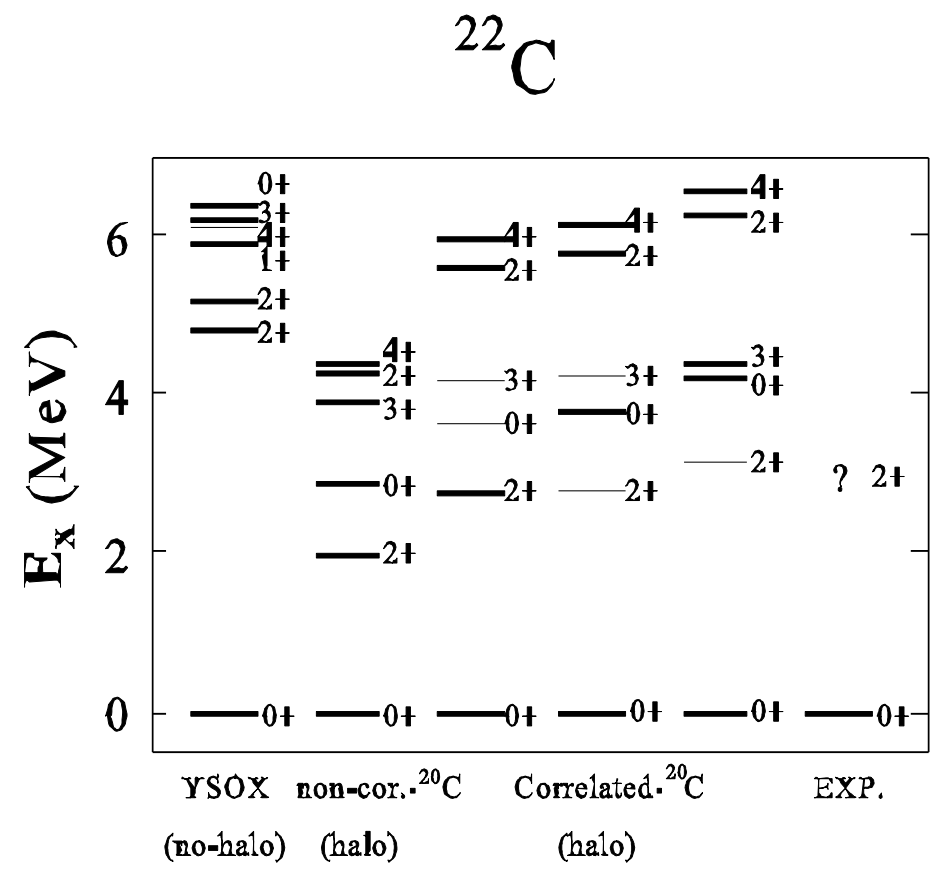

Fig. 2 Energy spectra of ${ }^{22} \mathrm{C}$ obtained by shell-model calculations with modified YSOX Hamiltonian. The first column is the case for the original YSOX Hamiltonian without the halo effects. Second column shows the result for the case without the correlation in ${ }^{20} \mathrm{C}$-core, which corresponds to $\beta=1$. The third, fourth and fifth columns are results with correlated-core. The value of $\beta$ decreases while the strength of the WS potential increases for more right side.

As $\beta$ becomes smaller with increasing strength of the WS potential, the energy levels of the excited states are pushed up. Occupation numbers of $2 \mathrm{~s}_{1 / 2}$ orbit in the ground state are 0.28 , 
0.24 and 0.18 , respectively, for the correlated $-{ }^{20} \mathrm{C}$ cases in Fig. 2. The case without the correlation with $\beta=1$ is also shown as "non-cor. $-{ }^{20} \mathrm{C}$ " where the occupation number of $2 \mathrm{~s}_{1 / 2}$ orbit is 0.94 . It would be quite interesting to measure the energy of the first $2+$ state. This can tell us information on how much $2 \mathrm{~s}_{1 / 2}$ component is blocked in the correlated ${ }^{20} \mathrm{C}$-core as well as $\mathrm{S}_{2 \mathrm{n}}$ from the strength of the WS potential.

\section{Strength of $n-n$ interaction and roles of three-body forces}

We compare the low-energy bare $n-n$ interaction with those in the medium. A Gaussian interaction which reproduces the scattering length and the effective range is used for the lowenergy limit of $n-n$ interaction, $V_{\text {low }}$. The expectation value of $V_{\text {low }}$ for the $2 s_{1 / 2}{ }^{2}(J=0)$ state evaluated for a harmonic oscillator (H.O.) wave function ( $\hbar \omega=14 \mathrm{MeV}$ ) is large in its magnitude compared with the matrix elements in conventional shell-model interactions in the medium. The matrix element $\mathrm{V}=\left\langle v 2 \mathrm{~s}_{1 / 2}^{2} ; \mathrm{J}=0\left|\mathrm{v}_{\mathrm{nn}}\right| v 2 \mathrm{~s}_{1 / 2}^{2} ; \mathrm{J}=0\right\rangle$ is shown in Fig. 3 for $\mathrm{V}_{\text {low }}$ as well as USD [20], USDA [21], USDB [21] and SDPF-M [22]. As we see from Fig. 3, the magnitudes for the shell-model interactions are smaller than that of $\mathrm{V}_{\text {low }}$ by $0.7-1.1 \mathrm{MeV}$. This difference can be attributed to the repulsion due to the effects of the three-body forces [23].

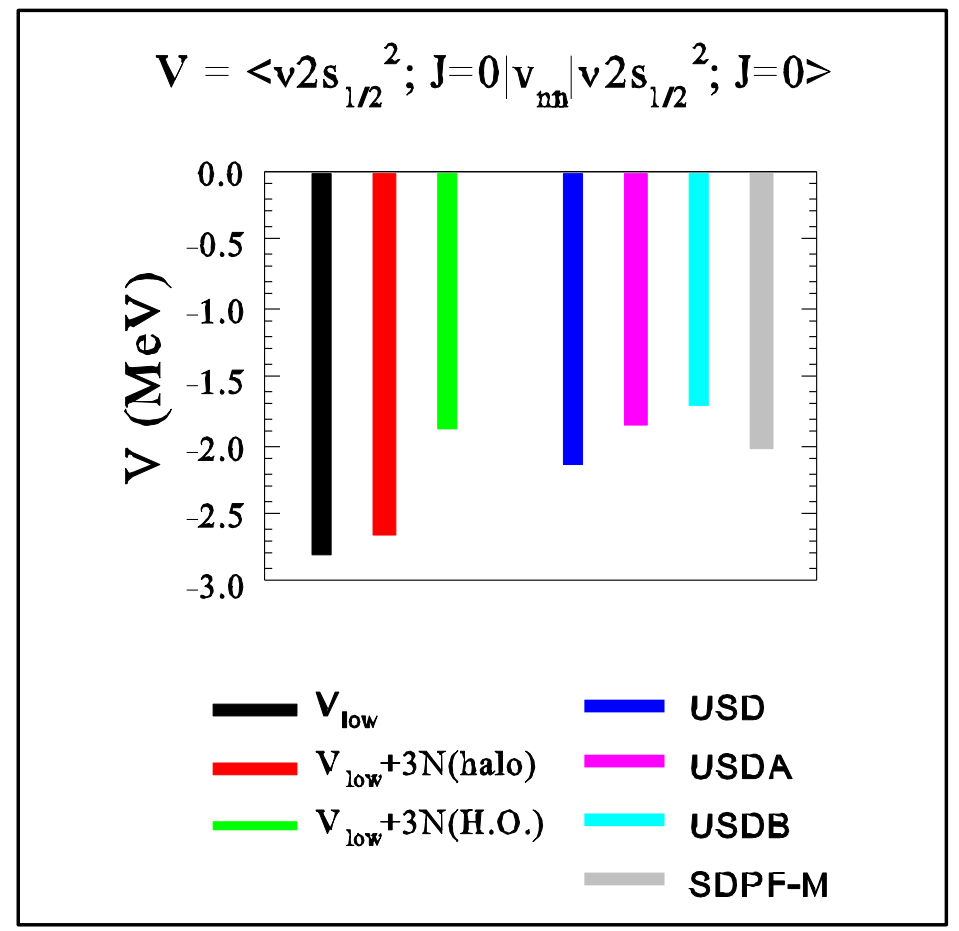

Fig. 3 Matrix elements of $\mathrm{v}_{\mathrm{nn}}$ for the $2 \mathrm{~s}_{1 / 2}{ }^{2}(\mathrm{~J}=0)$ state evaluated for various interactions; $\mathrm{V}_{\text {low }}$, $\mathrm{V}_{\text {low }}$ with three-body forces, and shell-model interactions, USD [20], USDA [21], USDB [21] and SDPF-M [22]

Contributions from the three-body forces are taken into account with the use of FujitaMiyazawa force which is induced by $\Delta_{33}$-isobar excitations through two-pion exchanges. The effective two-body matrix element for the valence neutron s-orbits is obtained by folding the ${ }^{20} \mathrm{C}$ core. The repulsive contributions from the three-body force reduce the attraction from $\mathrm{V}_{\text {low. }}$. The magnitude of the matrix element with the inclusion of the three-body force gets close to the values of the shell-model interactions for the case for harmonic oscillator radial wave functions. When the halo wave function is used for the s-orbit, the three-body force contributions become 
as small as $0.15 \mathrm{MeV}$, which is almost negligible. Thus for the halo in ${ }^{22} \mathrm{C}$, $\mathrm{n}-\mathrm{n}$ interaction remains strong enough to form the two-neutron halo.

\section{Summary}

We studied the formation of two-neutron halo in light drip-line nuclei, ${ }^{24} \mathrm{O}$ and ${ }^{22} \mathrm{C}$, using $n-n$ interaction fixed by the low-energy limit of $n-n$ scattering. $S_{1 n}$ and $S_{2 n}$ in ${ }^{24} \mathrm{O}$ are found to be well reproduced by our three-body model. The structure of the ground state of ${ }^{22} \mathrm{C}$ is studied in two different approaches with a closed and a correlated ${ }^{20} \mathrm{C}$. A unique relation between $\mathrm{S}_{2 \mathrm{n}}$ and halo radius is presented. $\mathrm{S}_{2 \mathrm{n}}$ is constrained to be less than $0.8 \mathrm{MeV}(0.3 \mathrm{MeV})$ for the correlated (closed) core from the condition that ${ }^{21} \mathrm{C}$ is unbound. The two-neutron halo state is successfully obtained in ${ }^{22} \mathrm{C}$ while ${ }^{21} \mathrm{C}$ remains unbound in the present three-body model. The halo radius is obtained to be 6-7 fm for the correlated core, which is consistent with the recent measurement [17] but smaller than those estimated for the closed-core case as well as the previous measurement [14]. Thus, the inclusion of the correlation in the ${ }^{20} \mathrm{C}$ is essential to reproduce the recent experimental halo radius. Because of the existence of the upper limit of the halo radius, there are no excited bound states. The spectra for ${ }^{22} \mathrm{C}$ are also studied. The energy of the first $2^{+}$ state is pushed up as the s-state in the core loses more $2 \mathrm{~s}_{1 / 2}$ component. The position of the $2_{1}{ }^{+}$ state can be a good measure of the correlation of the ${ }^{20} \mathrm{C}$ and the value of $S_{2 n}$.

The strength of the present low-energy limit $n-n$ interaction is large compared with shellmodel interactions in the medium. This difference can be explained with the repulsive contributions from the three-body forces, but for halo states this contribution is small. There are, therefore, no inconsistencies between the $n-n$ interaction used here for halos and the interactions in th medium.

This work was supported by Grants-in-Aid for Scientific Research (20244022, 22540290, 15K05090). It was supported also in part by Priority Isuue on post-K computer (hp160211) and CNS-RIKEN joint project for large-scale nuclear structure calculations. NA would like to acknowledge the JSPS Invitation fellowship program for long term research in Japan at the Tokyo Institute of Technology. CY acknowledges support from the National Natural Science Foundation of China under Grant No. 11305272.

\section{References}

[1] I. Tanihata et al., Measurements of Interaction Cross Sections and Nuclear Radii in the Light p-Shell Region, Phys. Rev. Lett. 55 (1985) 2676.

[2] M. V. Zhukov, B. V. Danilin, D. V. Fedorov, J. M. Bang, I. J. Thompson, and J. S. Vaagen, Bound state properties of Borromean halo nuclei: ${ }^{6}$ He and ${ }^{11}$ Li, Phys. Rep. 231 (1993) 151.

[3] P. G. Hansen, A. S. Jensen and B. Jonson, Nuclear Halos, Ann. Rev. Nucl. Part. Sci. 45 (1995) 591.

[4] W. Horiuchi and Y. Suzuki, ${ }^{22}$ C: An s-wave two-neutron halo nucleus, Phys. Rev. C 74 (2006) 034311 .

[5] M. T. Yamashita et al., Constraints on two-neutron separation energy in the Borromean ${ }^{22}$ C nucleus, Phys. Lett. B 697 (2011) 90. 
[6] Y. Kucuk and J. A. Tostevin, Intermediate energy four-body breakup calculations for ${ }^{22}$ C, Phys. Rev. C 89 (2014) 034607.

[7] B. Acharya, C. Ji and D. R. Phillips, Implications of a matter-radius measurement for the structure of Carbon-22, Phys. Lett. B 723 (2013) 196.

[8] N. Kobayashi et al., One- and two-neutron removal reactions from the most neutron-rich carbon isotopes, Phy. Rev. C 86 (2012) 054604.

[9] R. Machleidt, High-precision charge-dependent Bonn nucleon-nucleon potential, Phys. Rev. C 63 (2001) 024001;

G. A. Miller, M. K. Nefkens and I. Alaus, Charge symmetry, quarks and mesons, Phys. Rep. 194 (1990) 1 ;

C. R. Howell et al., Toward a resolution of the neutron-neutron scattering-length issue, Phys. Lett. B 444 (1998) 252;

D. E. Gonzalez Trotter et al., New Measurement of the ${ }^{1} S_{0}$ Neutron-Neutron Scattering Length Using the Neutron-Proton Scattering Length as a Standard, Phys. Rev. Lett. 83 (1999) 3788.

[10] NNDC, http://www.nndc.bnl.gov/nudat2/chartNuc.jsp

[11] A. Ozawa et al., Nuclear size and related topics, Nucl. Phys. A 693 (2001) 32.

[12] R. Kanungo et al., Exploring the anomaly in the interaction cross section and matter radius of ${ }^{23} \mathrm{O}$, Phys. Rev. C 84 (2011) 061304.

[13] L. Gaudefroy et al., Direct Mass Measurements of ${ }^{19} \mathrm{~B},{ }^{22} \mathrm{C},{ }^{29} \mathrm{~F},{ }^{31} \mathrm{Ne},{ }^{34} \mathrm{Na}$ and Other Light Exotic Nuclei, Phys. Rev. Lett. 109 (2012) 202503.

[14] K. Tanaka et al., Observation of a Large Reaction Cross Section in the Drip-Line Nucleus ${ }^{22}$ C, Phys. Rev. Lett. 104 (2010) 062701.

[15] C. Yuan, T. Suzuki, T. Otsuka, F. Xu and N. Tsunoda, Shell-model study of boron, carbon, nitrogen, and oxygen isotopes with a monopole-based universal interaction, Phys. Rev. C 85 (2012) 064324.

[16] T. Suzuki, T. Otsuka, C. Yuan and N. Alahari, Two-neutron "halo" from the low-energy limit of neutron-neutron interaction: Applications to drip-line nuclei ${ }^{22} \mathrm{C}$ and ${ }^{24} \mathrm{O}$, Phys. Lett. B 753 (2016) 199.

[17] Y. Togano et al., Interaction cross section study of the two-neutron halo nucleus ${ }^{22}$ C, Phys. Lett. B 761 (2016) 412.

[18] V. Efimov, Energy levels arising from resonant two-body forces in a three-body system, Phys. Lett. B 33 (1970) 563.

[19] A. Braaten and H.-W. Hammer, Universality in few-body systems with large scattering length, Phys. Rep. 428 (2006) 259.

[20] B. A. Brown and B. H. Wildenthal, Status of the nuclear shell model, Ann. Rev. Nucl. Part. Sci. 38 (1988) 29.

[21] B. A. Brown and W. A. Richter, New “USD” Hamiltonians for the sd-shell, Phys. Rev. C 74 (2006) 034315.

[22] Y. Utsuno, T. Otsuka, T. Mizusaki and M. Honma, Varing shell gap and deformation in N 20 unstable nuclei studied by the Monte Carlo shell model., Phys. Rev. C 60 (1999) 054315.

[23] T. Otsuka, T. Suzuki, J. D. Holt, A. Schwenk and Y. Akaishi, Three-Body Forces and the Limit of Oxygen Isotopes, Phys. Rev. Lett. 105 (2010) 032501. 